\title{
Anglais des biotechnologies santé à l'Université Grenoble Alpes pendant la crise sanitaire du printemps 2020
}

English for health biotechnology at Grenoble Alpes University during the spring 2020 health crisis

\section{Camille Biros et Laurence Matthys}

\section{CpenEdition}

\section{Journals}

Édition électronique

URL : http://journals.openedition.org/asp/6656

DOI : 10.4000/asp.6656

ISSN : 2108-6354

\section{Éditeur}

Groupe d'étude et de recherche en anglais de spécialité

\section{Édition imprimée}

Date de publication : 1 novembre 2020

Pagination : 65-73

ISSN : 1246-8185

\section{Référence électronique}

Camille Biros et Laurence Matthys, « Anglais des biotechnologies santé à l'Université Grenoble Alpes pendant la crise sanitaire du printemps 2020 », ASp [En ligne], 78 | 2020, mis en ligne le 01 novembre 2020, consulté le 06 mars 2021. URL : http://journals.openedition.org/asp/6656 ; DOI : https://doi.org/ $10.4000 / a s p .6656$

Ce document a été généré automatiquement le 6 mars 2021

Tous droits réservés 


\title{
Anglais des biotechnologies santé à l'Université Grenoble Alpes pendant la crise sanitaire du printemps 2020
}

\author{
English for health biotechnology at Grenoble Alpes University during the spring
} 2020 health crisis

Camille Biros et Laurence Matthys

\section{Avant-propos}

Après des mois de grèves interminables dans les transports publics, qui avaient déjà grandement perturbé les enseignements universitaires, l'annonce par le gouvernement français de la fermeture des universités le 17 mars 2020 à la suite de la pandémie de Covid-19 a forcé les enseignants à continuer à faire preuve d'adaptabilité. Même en dehors d'un contexte de crise, enseigner une langue de spécialité implique nécessairement de s'adapter à la terminologie, au public, ou encore aux pratiques pédagogiques propres au domaine, que l'utilisation de la plateforme SIDES (Système inter-universitaire dématérialisé d'évaluation en santé) illustre dans le cas présent. SIDES est une plateforme d'évaluation créée en janvier 2014 en vue de la passation des ECNi (Épreuves classantes nationales informatisées), qui correspondent au "nouvel Internat ». Commune aux trente-sept facultés de médecine, elle permet à chaque étudiant de s'entraîner librement sur des tests mis à sa disposition. Depuis quelques années, SIDES est utilisée également comme plateforme de formation, notamment en pédagogie inversée. L'enseignant peut alors créer des contenus, dont certains peuvent être autocorrectifs.

Dans le compte rendu d'expérience présenté ici, qui concerne l'adaptation d'un enseignement hybride d'anglais à destination des étudiants en licence de biotechnologie santé à l'Université Grenoble Alpes, les deux auteurs proposent de répondre aux questions essentielles que pose le dispositif à distance et asynchrone, a fortiori lorsqu'il est mis en place dans un contexte de crise: comment enseigner et évaluer la compétence d'interaction orale? Comment gérer le caractère chronophage de l'évaluation de la production orale? Comment conserver le lien entre enseignant et apprenant? Quel degré d'investissement peut-on exiger des apprenants? À quelle qualité 
peut-on s'attendre dans les travaux rendus? Comment éviter les fraudes éventuelles inhérentes à l'absence de surveillance lors des évaluations finales?

Elles y soulignent également la richesse de la classe inversée, la puissance des nouveaux outils tels que Wooclap, et l'utilité des productions recueillies pour la constitution de corpus d'apprenants. Enfin, leur inquiétude conclusive nous concerne tous et toutes au premier chef: le succès d'un tel dispositif ne risque-t-il pas de signer la fin de l'enseignement présentiel, indispensable, s'il est encore besoin de le rappeler, s'agissant des langues? (Pascaline Faure, Sorbonne Université)

\section{La transformation d'un enseignement hybride en enseignement à distance}

4 La crise sanitaire du printemps 2020 a imposé l'adoption d'outils d'enseignement à distance à une échelle inédite jusqu'alors. Il a fallu trouver des solutions dans l'urgence et essayer de les adapter à chaque contexte d'enseignement, notamment dans le domaine des langues de spécialité. Le contexte que nous souhaitons décrire concerne l'anglais pour la licence biotechnologie santé de la première année à la troisième année. Le nombre d'étudiants total inscrits dans ce parcours est de 300 (140 en L1, 80 en L2 et 80 en L3) auquel une équipe de quatre enseignantes dispense des enseignements pour valider trois crédits ECTS. Depuis la rentrée 2019, tous ces enseignements sont proposés en formule d'enseignement hybride (Biros \& Matthys 2019) avec une proportion d'enseignement en ligne variant selon les parcours, comme nous le détaillons dans le tableau 1 ci-dessous. En L2 et L3, les séances en présentiel sont hebdomadaires; en L1 elles ont lieu tous les quinze jours. Nous travaillons avec des groupes de 15 à 20 étudiants.

Tableau 1 : Distribution des volumes horaires (équivalent TD) des trois années de licence

\begin{tabular}{|l|l|l|}
\hline Parcours & En ligne & En présentiel \\
\hline L1 & 20 & 10,5 \\
\hline L2 & 20 & 24 \\
\hline L3 & 20 & 20 \\
\hline Total en Licence & 60 & 54,5 \\
\hline
\end{tabular}

5 Ce mode hybride d'enseignement, qui était relativement nouveau pour nous, a facilité notre travail pendant la crise sanitaire puisqu'il nous fournissait déjà un espace de travail en ligne que les enseignants et les étudiants avaient l'habitude d'utiliser (avec plus ou moins d'aisance et de réticence). Comme pour tous les enseignements en ligne des facultés de médecine et de pharmacie à Grenoble, nous utilisons la plateforme SIDES (Système inter-universitaire dématérialisé d'évaluation en santé1) qui, comme son nom l'indique, a été conçue au départ dans une optique d'évaluation mais dont les fonctionnalités sont régulièrement enrichies et permettent aussi de développer des ressources formatives. 


\section{Objectifs d'enseignement et d'apprentissage}

L'objectif principal de nos cours est de familiariser les étudiants avec le vocabulaire, les structures grammaticales et les genres de discours dont ils auront besoin dans leur futur cadre professionnel. Pour la mise en place de notre enseignement hybride, nous avons commencé par un bilan d'analyse de besoins de nos étudiants dont nous présentons les grandes lignes ci-dessous. Selon l'OCDE, la biotechnologie peut être définie comme "l'application de la science et de la technologie à des organismes vivants, de même qu'à ses composantes, pour modifier des matériaux vivants ou non vivants $\rrbracket^{2}$. Elle peut être utilisée à différentes fins, mais les étudiants de notre licence ne sont concernés que par les objectifs de santé et se destinent souvent à travailler dans l'industrie pharmaceutique, les laboratoires de recherche ou les hôpitaux. Ce sont des secteurs dans lesquels l'anglais est une langue de travail essentielle et les étudiants ont besoin de maîtriser aussi bien les compétences de compréhension et expression orales et écrites que la compétence d'interaction orale. Nous avons choisi d'évaluer les étudiants plutôt sur des compétences écrites en L1 et L2 et plutôt sur des compétences orales en L3. En effet, d'après les questionnaires de rétroaction que nous leur soumettons chaque année, ils se sentent souvent plus à l'aise à l'écrit et nous installons ainsi un principe de progressivité. Cependant, notre programme inclut un travail régulier sur les quatre compétences. Les enseignements sont organisés autour de quatre séquences thématiques par année, sélectionnées avec nos collègues spécialistes du domaine disciplinaire de nos étudiants. Pour chaque séquence, un point de langue, rencontré à plusieurs reprises dans les documents authentiques sélectionnés, est travaillé plus en détail.

7 Le contenu en ligne existant déjà sur SIDES comporte une ou plusieurs vidéos sur le thème choisi, assorties d'un questionnaire de compréhension, un ou deux articles (presse ou recherche) complétés par des questions de compréhension ou des exercices de vocabulaire, un SCORM (Shareable Content Object Reference Model) sonorisé qui traite du point de langue, incluant souvent une simulation de dialogue pour la mise en contexte des points étudiés. Des exercices de vocabulaire, de compréhension et de grammaire de type $\mathrm{QCM}$ et $\mathrm{QROC}$ (Question à réponse courte) permettent d'avoir un premier retour sur les compétences des étudiants pour ce qui est de la compréhension orale et écrite ainsi que sur des points précis d'expression écrite (utilisation de vocabulaire spécialisé en contexte, connaissances d'un point de langue spécifique). Pendant les TD d'une heure trente, nous commençons en général par une reprise des éléments essentiels vus en ligne et des erreurs principales effectuées dans les exercices sur la plateforme. Puis les étudiants sont répartis en petits groupes afin de travailler sur différents types de mises en situation où ils pratiquent l'expression et l'interaction orale en réutilisant les contenus vus en ligne (jeux de rôle, jeux de vocabulaire type tabou, débats), selon le principe de la classe inversée (Hoskins 2018; Lee \& Wallace 2018). Précisons que, depuis cette année, nous avons la chance de bénéficier de salles qui favorisent l'interactivité et le travail en petits groupes, avec du mobilier adaptable, des tableaux blancs sur tous les murs et la possibilité de projeter plusieurs contenus en même temps grâce à des tablettes. La plupart de nos TD ont lieu dans ces salles de cours qui favorisent l'interactivité et le travail de groupe. La question majeure à laquelle nous avons été confrontées pendant la crise sanitaire a été la suivante : comment conserver 
le principe d'interactivité et de pratique étudiante qui est au cœur de nos TD? Le contenu de nos enseignements est déjà en ligne; ainsi intégrer des diaporamas sonorisés et des contenus supplémentaires ne permettait pas de répondre à nos objectifs d'enseignement et d'apprentissage. Une autre question centrale au vu des effectifs étudiants et enseignants était la suivante : comment assurer un suivi pour un nombre si élevé d'étudiants tout en évitant de demander à notre équipe enseignante, également confrontée aux difficultés liées à la crise sanitaire, un effort disproportionné ? Les exemples que nous présentons dans les parties suivantes sont tous issus de l'enseignement dispensé en L3, mais les principes appliqués ont été les mêmes en L1 et L2.

\section{Pratiques pédagogiques}

\subsection{Structurer les contenus}

Afin de ne pas perturber le déroulé prévu de nos enseignements, la première étape pour la conversion des TD en contenu en ligne a été de s'appuyer sur l'objectif principal de chaque cours initialement prévu en présentiel. Nous avons cherché à remplacer les activités prévues en classe par des activités en ligne répondant au même objectif ou à un objectif proche. Nous avons dû adapter l'objectif surtout pour ce qui est de la compétence d'interaction orale que nous avons trouvée particulièrement difficile à faire travailler aux étudiants à distance avec des effectifs relativement importants. Pour maintenir de l'interaction, un travail sur des documents partagés, des forums et l'application Wooclap ${ }^{3}$ nous a semblé une bonne solution même si nous travaillions alors l'interaction écrite. Pour la production orale, l'envoi de fichiers audio individuels par les étudiants nous a semblé fonctionner. Les solutions de visioconférences pour de grands groupes montrent leurs limites lorsque le but est d'interagir, car dès que plusieurs personnes prennent la parole en même temps, le son est saccadé. Nous pensons qu'elles peuvent être utilisées de façon efficace avec des fonctionnalités avancées du type répartition des étudiants en petites salles virtuelles où l'enseignant peut circuler. Cependant, les applications de visioconférence mises à disposition des enseignants dans notre université ne nous ont pas permis d'expérimenter ce type d'outils. De ce fait, nous avons dû revoir nos objectifs d'enseignement pour ce qui est de l'interaction orale. Sur la plateforme, pour chaque TD, nous avons mis en ligne un dossier intitulé de la date à laquelle il était prévu. Nous y avons rassemblé tous les documents et les liens nécessaires pour ces activités. Nous mettions en ligne ces contenus quelques jours avant la date prévue afin que les étudiants puissent s'organiser à l'avance. Dans chaque dossier de TD, le premier document était un sommaire de cours contenant trois sections : 1- Objectif du TD, 2- Activités à faire par les étudiants, 3Retours à attendre des enseignants. Les étudiants pouvaient ainsi prendre connaissance de notre objectif, suivre les activités telles qu'elles étaient détaillées sur la fiche puis avoir des informations concernant les corrections qu'ils recevraient sur leur travail.

\subsection{Maintenir le lien avec les étudiants}

9 La crise sanitaire a souvent été la cause de situations personnelles difficiles pour les étudiants : confinement dans un petit logement, retour dans un environnement familial peu propice aux études, horaires de travail décalés ou augmentés, difficultés de 
connexion et pertes de lien social (Iribarnegaray 2020). Dans ce contexte, il nous a semblé essentiel de maintenir le lien avec nos étudiants autant que possible. Habituellement, en dehors des cours, nos interactions avec les étudiants se font plutôt de façon individuelle, par courriel. Durant cette crise, nous avons jugé nécessaire de mettre en place des forums en ligne pour chaque séquence. Ainsi, les étudiants avaient tous accès aux mêmes informations, chacun bénéficiant des réponses aux questions des autres, comme cela aurait eu lieu en classe. Nous avons assuré un suivi régulier de ces différents forums pour chaque unité d'enseignement. De plus, les étudiants bénéficiaient d'un retour enseignant sur leur travail dans tous les TD. Comme de nombreux étudiants nous faisaient part de leurs difficultés de connexion à certains moments de la journée, ou de leur indisponibilité sur les créneaux habituels, nous avons privilégié des outils de travail asynchrones. Ainsi, les étudiants pouvaient travailler sur nos ressources aux horaires et au rythme qui leur convenaient. Ce point, en plus de la non-conformité des outils de visioconférence gratuits avec le règlement général de protection des données (RGDP) et des difficultés à maintenir un niveau d'interactivité acceptable en grands groupes, nous a incitées à ne pas utiliser des outils de visioconférence en synchrone avec nos étudiants.

\subsection{Préserver l'équipe enseignante}

$10 \mathrm{Au}$ début du confinement, nous avons beaucoup utilisé la fonction "Dépôts de travaux » de la plateforme en ligne. Selon les compétences à travailler, les étudiants déposaient des documents écrits ou sonores, enregistrés à partir de leurs téléphones ou de leurs ordinateurs. Ils bénéficiaient ensuite de corrections des enseignants sur leurs travaux par l'intermédiaire de la plateforme. Cependant, l'équipe enseignante s'est vite retrouvée dépassée par l'ampleur des travaux de correction nécessaires. Nous avons donc décidé de convertir le plus de contenus possible en exercices interactifs de type QROC et QCM. Pour chaque TD nous nous sommes ainsi interrogées pour distinguer les contenus et objectifs d'enseignements qui pouvaient être atteint avec ce type d'exercices de ceux où il était essentiel de maintenir une correction personnalisée. Bien que ces corrections automatiques n'offrent pas un retour aussi qualitatif, adoptées en complément d'autres travaux, elles présentent l'avantage d'offrir un retour immédiat aux étudiants sur leur travail tout en limitant le travail de correction nécessaire.

\subsection{Varier les approches}

11 Afin de maintenir le niveau de participation et d'intérêt des étudiants, il nous a semblé important de varier les contenus et les outils. La plateforme SIDES a été notre outil principal de travail, comme elle l'est en temps normal. Nous y avons déposé, en plus du programme en ligne déjà existant, toutes les ressources complémentaires nécessaires pour remplacer les TD : vidéos, articles, forums, liens vers des documents partagés, et nous y avons écrit la plupart de nos questionnaires interactifs. Nous avons également utilisé l'application Wooclap qui offre l'avantage de donner aux étudiants un accès aux réponses des autres étudiants en plus des réponses proposées par l'enseignant.

Pour donner une idée plus précise de la façon dont nous avons cherché à varier les supports, nous détaillons une séquence d'enseignement à destination des L3. Cette séquence, qui se répartit en temps normal sur trois TD d'une heure trente, s'appuie sur 
les fictions qui traitent des biotechnologies de santé (livres, films ou séries). Notre objectif est de familiariser les étudiants avec le récit, notamment l'utilisation des temps et des mots de liaison dans ce genre de discours. À travers les fictions abordées, nous leur demandons de s'interroger sur le caractère réaliste ou non des contenus scientifiques, en s'appuyant sur un ou plusieurs articles de recherche. Un des critères d'évaluation est l'utilisation de discours rapporté avec une gamme étendue de verbes. Les questions éthiques, que les fictions permettent souvent d'aborder, sont aussi discutées dans ce contexte. En ligne, les contenus à consulter en temps normal avant le TD par les étudiants sont les suivants: SCORM sonorisé sur la séquence narrative, exercices de type QROC sur les mots de liaison et les temps, exemples de fictions abordant la biotechnologie, vidéo sur le réalisme de films de science-fiction et questionnaire de compréhension.

Pour remplacer le premier TD de la séquence, nous avons travaillé à partir de la fiction Never Let Me Go écrite par Kazuo Ishiguro en 2005 sur le thème du clonage et du don d'organe. Nous avons commencé par un article de critique publié par le Guardian qui présente la fiction, suivie d'une évaluation de compréhension écrite de son contenu. Nous avons ensuite proposé un résumé du livre dans lequel les étudiants devaient identifier les différentes étapes de la séquence narrative: situation initiale, complication, action, résolution et situation finale. Nous avons ensuite mis à disposition des étudiants deux extraits filmés disponibles sur YouTube que nous avions intégrés à la plateforme avec des hyperliens. Ces extraits étaient complétés par des questionnaires de compréhension de type QCM. À partir d'extraits du livre, nous avons alors conçu des exercices de type QROC où les étudiants devaient rétablir les temps et mots de liaison. Pour remplacer le deuxième TD, nous avons abordé, à l'aide d'un sondage, les questionnements éthiques dont l'œuvre témoigne. En donnant des exemples de travaux étudiants des années passées, nous avons invité les étudiants à réfléchir à d'autres œuvres de fiction représentant leur domaine scientifique et à voir comment ils pourraient les raconter grâce à la structure de la séquence narrative présentée précédemment. Ils devaient également signaler au moins un article scientifique qu'ils utiliseraient pour aborder le caractère réaliste de l'œuvre et commencer à en résumer le contenu. Il s'agissait de travaux préparatoires en vue de l'oral final qui remplaçait le dernier TD.

14 Pour ce dernier, les étudiants devaient déposer en ligne un diaporama sonorisé ou une vidéo de huit minutes dans lesquels ils présentaient une œuvre de fiction de leur choix, racontaient de façon succincte sa structure narrative puis analysaient son caractère plus ou moins réaliste à la lumière d'articles scientifiques qu'ils citaient. Il s'agit d'un exercice que nous avons l'habitude de faire en cours lors de présentations orales classiques. Cependant, le fait de devoir déposer en ligne ces contenus a demandé une certaine adaptation des étudiants au format. Il leur a été possible de reprendre leurs erreurs, de rectifier leur texte et, dans l'ensemble, de produire des présentations d'une meilleure qualité que celles des années précédentes. Certains étudiants, qui maitrisaient bien les outils technologiques, ont intégré les images du film et de l'article scientifique qu'ils commentaient pendant qu'elles défilaient à l'écran. Dans l'ensemble, nous avons été impressionnées par la qualité des travaux déposés. Tous les étudiants ont pu rendre leur travail. Par ailleurs, grâce à la mise en ligne de ces contenus, nous disposons, dorénavant, de nombreuses présentations que nous pouvons garder pour 
analyser les fautes récurrentes et donner des exemples aux étudiants des années à venir.

\section{Modalités d'évaluation}

Si de nombreuses évaluations se font déjà en mode électronique à l'UFR de pharmacie de Grenoble, qui est équipée de tablettes dans ce but, la non-surveillance des examens pendant la crise sanitaire change les conditions d'évaluation. Les fraudes à distance sont très faciles pour les exercices de type $\mathrm{QCM}$ et nous avons donc inclus au moins un QROL (Question à Réponse Ouverte Longue) pour chaque examen. Comme détaillé précédemment, pour les L3 dont les compétences évaluées sont essentiellement orales, la présentation orale déposée en ligne constituait un élément essentiel d'évaluation. Nous avons également adapté le format des évaluations écrites qui étaient dans l'ensemble plus longues avec un temps plus limité que d'habitude afin d'éviter les possibilités de communication et de consultation des cours pendant l'examen. Nous nous sommes questionnées sur l'évaluation du travail fait en ligne par les étudiants. Dans les circonstances exceptionnelles de la crise sanitaire, tous les exercices en ligne ont été mis en mode auto-évaluation sans notes, et nous n'avons pas consulté les données concernant les temps de connexions et nombre d'accès afin de ne pas pénaliser les étudiants dont les problèmes de connexion avaient rendu difficile le travail régulier sur la plateforme d'enseignement.

\section{Bilan de l'action de formation}

Globalement, nous pensons pouvoir tirer un bilan positif de la continuité pédagogique mise en place dans le contexte de la crise sanitaire. Nos communications avec les étudiants ont été régulières; leur assiduité a été satisfaisante à l'exception d'une dizaine d'étudiants sur l'ensemble des promotions, la qualité des travaux rendus est bonne. Dans le questionnaire de satisfaction des L3 que nous soumettons chaque année aux étudiants, nous avons ajouté une question spécifique portant sur l'évaluation de notre enseignement pendant la crise. Il s'agissait d'une question avec un curseur où les étudiants pouvaient faire varier la note entre 0 (correspondant à qualité basse, « poor ») et 100 (correspondant à très bonne qualité, « very good»). Soixante-douze étudiants sur quatre-vingts ont répondu au questionnaire et la moyenne obtenue est de 89. Plusieurs étudiants ont pris le temps de nous remercier pour nos efforts pendant cette crise. Nous pensons que notre utilisation précédente de la plateforme pour l'enseignement en mode hybride a facilité notre approche. Nous avons quand même dû nous adapter à un contexte particulier et prendre en compte des situations personnelles compliquées, tant au niveau des étudiants que des enseignants, en offrant un cadre de travail souple. Nous avons été obligées d'expérimenter de nouveaux outils notamment les forums, les dépôts de travaux commentés, les documents collaboratifs, que nous n'avions pas eu l'occasion d'utiliser jusque-là.

17 À l'heure où nos institutions nous demandent d'anticiper une rentrée au moins partiellement à distance, l'expérience que nous avons eue ce semestre nous conforte dans notre capacité à faire face à cette situation. Grâce à un transfert de connaissances entre les membres de l'équipe enseignante, les compétences d'utilisation des différentes fonctionnalités des plateformes d'enseignement et d'applications 
sélectionnées ont été renforcées pour chacune. Nous espérons que notre apprentissage dans l'urgence ce semestre nous rendra plus aptes à nous adapter aux futures situations plus ou moins délicates auxquelles nous risquons tous d'être confrontés pour l'enseignement des langues de spécialité dans un horizon proche. La crise sanitaire a aussi été l'occasion de souligner à quel point l'enseignement hybride est appréciable lorsqu'il se fait de façon vraiment hybride. Le principe de la classe inversée nous semble riche de possibilités dans l'enseignement des langues parce qu'il permet aux étudiants d'être plus actifs et de travailler de façon interactive avec l'enseignant en classe, à partir de ce qu'ils ont déjà acquis en ligne. Il nous semble qu'il y a forcément une perte d'interactivité et de spontanéité dans les enseignements en ligne. Si le présentiel est réduit, l'intérêt de la classe inversée l'est aussi. Espérons que les efforts faits par les enseignants pour rester connectés avec leurs étudiants malgré la crise sanitaire ne seront pas utilisés comme un argument pour réduire encore ce qui fait le cœur de notre métier et ce qui reste un moyen inégalé de rester en lien, la présence.

\section{BIBLIOGRAPHIE}

BIROS, Camille \& Laurence MATTHYS. 2019. "Designing a hybrid course in English for Biotechnology for Health Students in Grenoble Alpes University". ICERI 2019 Proceedings, 1795-1802.

HOSKINS, Laura. 2018. "Course design for first-year undergraduate Human Science programmes: A blended course in English for Academic Purposes". ASp 74, 173-189.

IRIBARNEGARAY, Léa. 2020. «Isolement, précarité, perte de repères : comment le confinement a fait basculer psychologiquement certains étudiants ». Le Monde, 26 mai 2020.

LEE, Given \& Amanda WALLACE. 2018. "Flipped learning in the English as a Foreign Language Classroom: Outcomes and perceptions". Tesol Quarterly 52/1, 62-84.

\section{NOTES}

1. <https://side-sante.fr $\geq$.

2. <http://www.oecd.org/fr/sti/tech-emergentes/ definitionstatistiquedelabiotechnologiemiseajour en2005.htm>, consulté le 20 janvier 2020. 3. <https://www.wooclap.fr>. 
INDEX

Mots-clés : anglais des biotechnologies, classe inversée, crise sanitaire

Keywords : English for biotechnology, flipped class, health crisis

\section{AUTEURS}

\section{CAMILLE BIROS}

Camille Biros est maître de conférences à la Faculté de médecine et de pharmacie de Grenoble (Université Grenoble Alpes) où elle est responsable du département d'anglais et enseigne l'anglais pour la médecine, la pharmacie et les biotechnologies. Ses recherches portent sur les discours, la terminologie et la traduction spécialisée dans les domaines de l'environnement et de la santé. camille.biros@univ-grenoble-alpes.fr

\section{LAURENCE MATTHYS}

Laurence Matthys est professeur certifiée à la Faculté de médecine et de pharmacie de Grenoble (Université Grenoble Alpes) où elle enseigne l'anglais pour la médecine, la pharmacie et les biotechnologies. Elle est responsable de l'enseignement d'anglais en master industrie pour la santé. laurence.matthys@univ-grenoble-alpes.fr 